\title{
Pertenencia ciudadana: estudio de caso de las comunidades afro de la ciudad de Cartagena de Indias, Colombia
}

\author{
María C. Bustillo-Castillejo', Yesid De La Espriella-Mendoza ${ }^{1}$ y Jhorquis Machado-Licona2* \\ (1) Facultad de Ciencias Económicas, Administrativas y Contables, Universidad Libre, Cartagena, Colombia \\ (correo-e: mariac.bustilloc@unilibre.edu.co; yesidi.delaespriellam@unilibre.edu.co). \\ (2) Facultad de Ciencias Contables y Administrativas, Corporación Universitaria Rafael Núñez, Cartagena, Colombia \\ (correo-e: jhorquis.machado@curnvirtual.edu.co)
}

* Autor a quien debe ser dirigida la correspondencia.

Recibido Oct. 27, 2020; Aceptado Dic. 30, 2020; Versión final Mar. 22, 2021, Publicado Ago. 2021

\begin{abstract}
Resumen
El presente estudio busca identificar cuáles son las variables que inciden en el pensamiento de las comunidades afro-descendientes de la ciudad Cartagena de Indias (Colombia) en torno a su identidad ciudadana y sentido de pertenencia. La comunidad afro-descendiente, es un segmento de las minorías poblacionales que ha sufrido cierto abandono histórico por parte del estado. La población estudiada está compuesta por la comunidad afro-descendiente de la ciudad de Cartagena de Indias y la muestra está constituida por líderes comunitarios de las diversas poblaciones raizales. El diseño es mixto con metodologías cuantitativas y cualitativas y se implementa la regresión logística. Como resultado se obtuvo que las variables que explican el modelo son: la participación a través de ser miembro de consejo comunitario, la gestión de los líderes comunitarios y la valoración cultural. Se concluye que, aún en abandono, los afro-descendientes se sienten ciudadanos cuando le dan valor a su cultura y espacios de participación.
\end{abstract}

Palabras clave: ciudadanía; comunidad; identidad cultural; participación democrática; liderazgo

\section{Citizenship and belonging: a case study of Afro-communities from the city of Cartagena de Indias, Colombia}

\begin{abstract}
The present study seeks to identify what variables influence civic identity and sense of belonging in Afrodescendant communities from the city of Cartagena de Indias (Colombia). The Afro-descendant community is a segment of a population minority that has suffered historical abandonment by the state. The population examined consists of Afro-community leaders representing their ancestral diversity. The methodology is mixed, qualitative and quantitative, and the logarithmic binary regression model is applied. The results show that the variables that best explain the model are participation through being a community council member, community leader's management, and cultural valuation. It is concluded that, even in abandonment, Afro-descendants feel they are citizens when they value their culture and their spaces for participation.
\end{abstract}




\section{INTRODUCCIÓN}

El concepto de ciudadanía esta intrínsecamente ligado al sentido de pertenencia, entendiendo este como la adquisición de derechos en tres esferas: la civil, ésta se da cuando se reconoce que todas las personas son libres, a través de la abolición de la esclavitud, la censura de prensa y la posibilidad de emanciparse de las creencias católicas; en la esfera política, al reconocer el derecho al sufragio para los diversos sectores poblacionales más allá de la sociedad formada por los terratenientes; y en lo social, cuando se comprende la comunidad como sociedad de derecho con la capacidad y el derecho de participar desde la política en la construcción del bien común. (Moses, 2019); en ese orden de ideas el concepto de ciudadanía vas más allá de un simple estatus, ya que implica el sentimiento de ser parte de la construcción social a través de la participación (Olivo, 2017). En Cartagena de Indias se han venido desarrollando fenómenos que dificultan la cohesión social y debilitan el sentido de pertenencia de los ciudadanos, especialmente en las comunidades étnicas objeto del presente estudio. Uno de los factores que afectan la cohesión social y por ende sentido de pertenencia, dado que genera exclusión es la pobreza.

Del tema de la pobreza se ha dicho mucho en la ciudad de Cartagena de Indias, sobre esto se puede referenciar a Espinosa-Espinosa et al., (2020) quienes señalan que entre las causas de la pobreza económica de la ciudad se encuentran: la herencia colonial que no ha permitido la dotación en términos de conocimiento y habilidades a las poblaciones más vulnerables principalmente la afrodescendientes; la poca capacidad de empleo formal por parte de la economía de la ciudad, esto debido principalmente a su estructura empresarial; la dualidad empresarial que se presenta por la convivencia de empresas formales e informales en donde las últimas no cumplen con los estándares necesarios para negociar con las formales; la alta concentración del ingreso y del capital en pocas manos; el estancamiento en la transformación productiva, esto se debe al crecimiento concentrado en pocos sectores lo que hace evidente la necesidad de dar el salto hacia otros productos de valor agregado; no hay suficientes empresas en la ciudad para generar más empleo; poco uso de la ciencia, la tecnología y la innovación; emprendimientos poco sostenibles; la desarticulación y la crisis con el campo especialmente el sector agropecuario, la recepción de la población migrante o desplazada, la debilidad en la oferta laboral o talento humano, y por último la debilidad institucional lo que se observa por la falta de voluntad para el desarrollo de una política pública eficiente.

Por su parte, Ayala-García y Meisel-Roca (2017), señalan que a pesar de ser Cartagena de Indias una de las ciudades de Colombia que más aporta al Producto Interno Bruto (PIB), es de las que presenta mayores índices de pobreza y desigualdad, ello es observable mediante indicadores como: la pobreza monetaria que está alrededor del $30 \%$ y la pobreza extrema que ronda los seis puntos porcentuales muy lejos de cifras más favorables observadas en otras ciudades de Colombia. Cabe aclara que la pobreza extrema es un fenómeno multidimensional que implica: el nivel de ingreso, la calidad de vivienda, el acceso a servicios de salud, educación y empleo digno. En materia de exclusión el panorama de Cartagena de Indias no es distinto a lo anteriormente planteado, ya que la ciudad ocupa el segundo lugar entre las trece principales ciudades de Colombia con mayor índice de exclusión social.

El fenómeno de la pobreza en la ciudad de Cartagena muestra mayor acentuación en las zonas donde se encuentran ubicadas las comunidades étnicas que se están analizando. Estas zonas se caracterizan por la ausencia de los servicios público básicos (acueducto, alcantarillado, gas, vías y fluido eléctrico), condiciones de vivienda poco adecuadas, ausencia de servicios de salud, altos niveles de analfabetismos, desempleo, hacinamiento, empleo informal y en algunos casos trabajo infantil. Estas son condiciones que no generan cohesión social, fortaleciéndose de este modo la exclusión social que afecta el sentido de pertenencia y el ejercicio de la ciudadanía.

Desde una perspectiva histórica, Barreira et al., (2013) establecen que el imaginario de ciudadanía en Colombia que se réplica en Cartagena, está ligado al conflicto que se desarrolla entre los sectores populares o emergentes por ser incluidos socialmente y las elites que no permiten la inclusión social. Los autores sostienen que, en Cartagena de Indias, los criterios de procedencia de la persona, el grupo racial y la clase social, siguen siendo utilizados para discriminar y excluir a las minorías, entre ellas los indígenas y afrodescendientes. El efecto de la discriminación recibida por los ciudadanos principalmente los afrodescendientes genera en dicho segmento poblacional impactos en diversos ámbitos como lo político ya que existen pocos espacios de participación en la toma de decisiones para la construcción de la ciudad, lo educacional, en la capacidad de empleabilidad, en lo cultural y principalmente en lo relacionado con la salud pública. (Lennox y Minott, 2011). Este estado de "invisibilidad social", ha llevado en muchos casos al desconocimiento de la contribución de las comunidades afrodescendientes en la historia y la cultura, afectando su sentido de pertenencia y generando en estos la percepción de ser ciudadanos de segunda clase. (Hooker, 2008) 
Para Fonseca et al., (2018) la cohesión social es un constructo que debe ser materializado mediante la convivencia y aceptado la diferencias entre los ciudadanos. El estudio de Corpovisionarios (2009) que versó sobre el diagnostico de cultura ciudadana en Cartagena de Indias, puso de manifiesto la poca cohesión social en Cartagena de Indias caracterizada por la inequidad, la fragmentación y la exclusión social. Esto se ve reflejado en los resultados del estudio que arroja desigualdades sociales en la apropiación del espacio público sobre todo en el centro histórico, falta de integración social en el transporte, desigualdades sociales en el ejercicio de la ciudadanía, altos niveles de desconfianza en los acuerdos y en las instituciones, mucha inseguridad sobre todo en espacios públicos, violencia intrafamiliar, y problemas de convivencia y tolerancia. Todo lo anterior aunado a una situación alta pobreza, dificultad en el acceso y permanencia en el sistema educativo de las comunidades más vulnerables, la imposibilidad de algunas comunidades de asistir a actividades culturales programadas en la ciudad, la alta diferenciación entre los diferentes estratos sociales y la ausencia de espacios de integración para los miembros de la comunidad, constituyen un panorama poco alentador que afecta el sentido de pertenencia.

Otro de los elementos que refuerzan el sentido de pertenencia es la participación ciudadana, esta implica el involucramiento de los ciudadanos en la toma de decisiones que los afectan. Mediante la participación ciudadana se fortalece la democracia, refuerza las habilidades cívicas ya que fomenta la inclusión (Michels y De Graaf, 2017). Bherer et al., (2016) coinciden al afirmar que la participación ciudadana es observada como un mecanismo cuya función es permitir la inclusión de los aportes de los ciudadanos en las decisiones públicas. La sociedad Cartagenera se caracteriza por un alto índice de conflicto social, sobre todo del estado con las comunidades afrodescendientes, el ejercicio de la participación ciudadana desde lo político se constituye en una herramienta fundamental para la búsqueda de soluciones a las disputas que se presentan sobre todo por el territorio. Es importante que el estado ceda poder frente a las iniciativas de la ciudadanía (Ortiz, 2012), con el fin de generar en la conciencia de los ciudadanos la existencia de un gobierno abierto a la escucha de las propuestas ciudadanas, que apoyen la construcción de una nueva realidad social (Wirtz et al., 2018)

Un instrumento empleado por las comunidades afro descendientes mediante el cual estas pueden ejercer la participación ciudadana es la participación comunitaria, la cual se puede comprender como un mecanismo que le otorga a las comunidades el poder de negociar con actores públicos-privados en torno a la toma de decisiones que los afectan (Hyland y Bertsch, 2018). A través la participación comunitaria se consolida la identidad colectiva del grupo, ya que cada uno de los integrantes del colectivo ven satisfechas sus inquietudes y necesidades, lo cual los hace sentirse más parte de un grupo, fortaleciéndose de esta manera la participación, y logrando en conjunto con otros miembros del grupo la transformación social (Graizbord, 1999).

Un concepto no menos importante para esta investigación, lo constituye el liderazgo comunitario como el elemento que va a permitir consolidar todas aquellas iniciativas que las comunidades decidan desde lo colectivo. El liderazgo comunitario se convierte en puente que dinamiza la calidad de vida de los ciudadanos, por ser el medio de negociación de los mismos con las organizaciones (Xu et al., 2017). Dentro de este espectro, el papel del líder comunitario es transcendental, dado que este es el encargado de transferir los intereses entre las partes (comunidad y estado) (Martiskainen, 2017), de esta manera se convierte en el vocero de la comunidad que defiende y a quien tiene que rendir cuentas. Uno de los aspectos que concita un gran interés por parte de las comunidades afrodescendiente está relacionado con la protección de sus derechos como comunidad ancestral, históricamente estas comunidades han sufrido la vulneración de sus derechos, por medio del desplazamiento de las tierras que por años han habitando. Frente a este hecho, el estado colombiano ha desarrollado una serie de normativas para evitar el continuo desarraigo de estas comunidades, estos desarrollos reposan en la constitución política colombiana, y en otra serie de leyes como la ley 99 de 1993 y la ley 10 del mismo año, y en sentencias proferidas por la honorable Corte Constitucional colombiana.

Esta normatividad busca garantizar que, a través de mecanismos como la consulta previa, se protejan los derechos de los pueblos raizales sobre los territorios y sus recursos naturales, por encima de los intereses de terceros. La consulta previa facilita el reconocimiento de la identidad cultural de los pueblos afrodescendientes originarios, dando la posibilidad de lograr su desarrollo social y económico que les permita salir de la trampa de la pobreza que por mucho tiempo han padecido. La materialización de la consulta previa en la población afrocolombiana desarrolla el derecho que esta tiene de conservar y reforzar sus instituciones y de participar plenamente en las decisiones que atañen a su vida política, económica y cultural. Para hacer efectivo este derecho es necesario: primero, identificar las comunidades donde se realizará la consulta; segundo, identificar los actores representativos en cada comunidad; tercero, garantizar el suficiente tiempo para cada proceso de consulta; y cuarto, asegurar una adecuada respuesta del gobierno a las decisiones de la población afrocolombiana. Aunque la consulta previa como mecanismo de participación comunitaria existe y es acogida por los pueblos raizales para la defensa de sus territorios y de sus derechos adquiridos, en muchos casos es una herramienta que no asegura por completo la soberanía de los pobladores sobre sus recursos naturales y 
culturales, esta afirmación deriva de los diversos conflictos legales que se presentan en los territorios, sin embargo la gran bondad de la consulta previa radica en su importancia para la generación de espacios de participación (McNeish, 2017).

\section{METODOLOGÍA}

Para el desarrollo del presente estudio se implementan características de las metodologías cuantitativas y cualitativos por lo cual la investigación tiene un diseño mixto. Las metodologías mencionadas suelen contrastarse y complementarse, siendo la metodología cuantitativa la que aporta los datos fácticos o duros, observando la realidad desde la visión objetiva, pero dejando de lado datos necesarios para una mejor comprensión de los fenómenos, por su parte a la metodología cualitativa se le otorga un carácter interpretativo o subjetivo sin embargo esta desarrolla una perspectiva más profunda que captura datos que los investigadores no previeron. Las diferencias presentes entre ambas metodologías se complementan enriqueciendo el desarrollo de las investigaciones (Barnham, 2015).

Para Schick-Makaroff et al., (2016) la metodología cuantitativa posee un criterio bien establecido, lo que conlleva a la correcta comprensión y plena identificación de los elementos integrantes del problema investigado, así como el alcance del mismo, el impacto y la relación existente entre las variables que lo componen. Una herramienta clave en la investigación cuantitativa es la estadística, mediante esta se puede analizar el comportamiento de una muestra tanto en el nivel descriptivo como en el nivel inferencial, permitiendo este último nivel deducir las variables de mayor incidencia en un fenómeno estudiado, logrando incluso proponer y validar modelos que explican cómo se interrelacionan o se agrupan las variables. Lo anterior permite afirmar que la estadística facilita las herramientas que ayudan a los investigadores a desarrolla estudios con resultados objetivos. Bolondi et al., (2018)

Para comprender la naturaleza metodológica del presente estudio, es importante indicar su objetivo el cual es identificar las variables de mayor incidencia en el sentido de pertenencia de las comunidades afro descendientes de la Ciudad de Cartagena de Indias, por lo anterior, la investigación emplea la técnica de estudio de caso, que de acuerdo con (Yin, 2013) es una estrategia para el desarrollo de las investigaciones en ciencias sociales, mediante la cual se puede indagar el "cómo" y el "por qué" se da una determinada problemática. En la actualidad el estudio de casos es una de las técnicas más implementadas por investigadores en los distintos niveles, ello se debe a que facilita el análisis de los factores centrales de los fenómenos a la vez que ayuda a la correcta y amplia caracterización de los mismos dentro de un alcance plenamente establecido.

Otra razón que justifica la amplia utilización de los estudios de caso es explicada por (Eisenhardt, 1989) ya que indica que el estudio de caso es una estrategia que facilita entender los fenómenos altamente cambiantes de la modernidad. En la naturaleza del estudio de caso se hallan: la transversalidad, lo que facilita implementarlo en diversas áreas de conocimiento (educación, política y sociología, entre otras) cubriendo un amplio rango de las llamadas ciencia sociales; la versatilidad es tal que ayuda al abordaje de diversos casos desde distintas perspectivas (Harrison et al., 2017); en consonancia con su versatilidad los estudios de caso aportan un gran número de fuentes mixtas para la recogida de datos (entrevistas, grupos focales, observación directa) (Chetty, 1996). Los estudios de caso coadyuvan en el abordaje a profundidad de fenómenos específicos, ayudando a las investigaciones sin precedentes a la identificación de variables dependientes y formulación de hipótesis previas. Meredith et al., (1989)

Como parte del análisis cuantitativo se utilizó la regresión logística, definida por Lever et al., (2016) como un método estadístico muy utilizado para fenómenos de estudios donde la variable dependiente asume valores en un conjunto finito. Mansournia et al., (2018) coincide con Lever et al., (2016) al indicar que la regresión logística es un método de la estadística que facilita la validación de las contribuciones de los diferentes elementos que aportan a la comprensión de un fenómeno; siendo esta mayormente empleada cundo existen variables que poseen una capacidad politómica (nivel de acuerdo, frecuencia, nivel de importancia, valoración, probabilidad).

En la presente investigación se implementó la regresión logística binaria por medio del programa SPSS de IBM. La población estudiada fueron las comunidades afrodescendientes de la Ciudad de Cartagena de Indias y la muestra estuvo constituida por líderes comunitarios de las diversas poblaciones raizales, para la selección de los líderes se tomó a consideración el muestreo por conveniencia. De otra parte, dentro de las variables del estudio se encuentran: ser ciudadano de Cartagena de Indias (variable dependiente) y garantes de derechos, participación comunitaria, gestión del liderazgo comunitario, valoración de la cultura, participación democrática e identidad afrodescendiente (variables independientes) (Tabla. 1), por medio de estas variables se pretender resolver la hipótesis nula $\mathrm{H}_{0}$, la cual indica que la probabilidad ocurrencia de que las personas se sientan ciudadanos de Cartagena no se puede predecir por las variables independientes 0 validar la 
hipótesis alternativa $\mathrm{H}_{1}$, por la cual la probabilidad de sentirse ciudadano si puede predecirse por las variables independientes descritas.

Para el diseño del instrumento (encuesta) se tomaron los conceptos de desarrollo social, e índice de progreso social, en los cuales se determinan tres dimensiones: necesidades, oportunidades y bienestar, y progreso y organización, finalmente en esta última dimensión de progreso se anclan las variables de relacionamiento comunitario, cohesión y liderazgo comunitario que incluye el tema de sentido de pertenencia. Otro elemento que caracterizo el instrumento es que contó con 15 preguntas asociadas con las variables que se muestran en la operacionalización de las variables (Tabla 1); la estructura del instrumento permitió un mejor acercamiento a los rasgos culturales de las comunidades afrodescendientes, abordando de este modo elementos como su estructura comunitaria, su comprensión sobre los espacios de participación, su forma de aplicación de liderazgo y su concepción de ser ciudadano.

Tabla 1: Operacionalización de variables.

\begin{tabular}{|l|l|}
\hline \multicolumn{2}{|c|}{ Variables } \\
\hline Dependiente & Ciudadano de Cartagena de Indias \\
\hline Independientes & Identidad afrodescendiente \\
\cline { 2 - 2 } & Garantes de derechos \\
\cline { 2 - 2 } & Participación comunitaria \\
\cline { 2 - 2 } & Gestión del liderazgo comunitario \\
\cline { 2 - 2 } & Valoración de la cultura \\
\cline { 2 - 2 } & Participación democrática \\
\hline
\end{tabular}

Por último, la tabla 2 muestra los resultados de la validación del instrumento, para ello se empleó el coeficiente de alfa de Cronbach el cual es una herramienta para verificar la consistencia interna (Campos-Carreño et al., 2020). Como se puede observar en la tabla 2 el coeficiente obtenido es de 0,655 , lo que indica que el instrumento (encuesta), tiene un nivel de fiabilidad adecuado, logrando de este modo medir el fenómeno estudiado a través de las variables propuestas.

Tabla 2: Validación del instrumento (alfa de Cronbach)

\begin{tabular}{|c|c|c|c|}
\hline \multicolumn{4}{|c|}{ Resumen de procesamiento de casos } \\
\hline \multirow{2}{*}{ Casos } & Válido & $\mathrm{N}$ & $\%$ \\
\cline { 2 - 4 } & Excluido $^{\mathrm{a}}$ & 1 & 99,0 \\
\cline { 2 - 4 } & Total & 98 & 1,0 \\
\hline a. La eliminación por lista se basa en todas las variables del procedimiento. \\
\hline \multicolumn{3}{|c|}{ Estadísticas de fiabilidad } \\
\hline \multirow{2}{*}{ Alfa de Cronbach } & $\begin{array}{c}\text { Alfa de Cronbach basada en } \\
\text { elementos estandarizados }\end{array}$ & $\mathrm{N}$ de elementos \\
\hline 0,655 & \multicolumn{2}{|c|}{0,738} \\
\hline
\end{tabular}

\section{RESULTADOS Y DISCUSIÓN}

Después de incluir los datos en el modelo a través de la regresión logística binaria, la ocurrencia de que los miembros de comunidades de las minorías étnicas afrodescendientes tengan sentido de pertenencia como ciudadanos de la ciudad de Cartagena de Indias, a pesar de los rezagos históricos que los llevan al pobre desarrollo en sus territorios, frente a los fenómenos causales de las variables independientes: identidad, cultura, participación, derechos y liderazgo comunitario. Se describe la posibilidad de ocurrencia de que las comunidades afro descendientes se sientan cartageneros con el desglose de la hipótesis alternativa $\mathrm{H}_{1}$, en seis sub hipótesis con la finalidad de identificar a ciencia cierta cuáles son las variables independientes que le realmente tienen efecto causal para la hipótesis alternativa. (Tabla 3). El resumen incluyendo los datos procesados por el modelo, los casos perdidos, los casos no seleccionados y su codificación se presenta en la Tabla 4. 
Tabla 3: Desglose de la hipótesis alternativa $\mathrm{H1}$

\begin{tabular}{|c|l|}
\hline Sub hipótesis & \multicolumn{1}{c|}{ Enunciado } \\
\hline $\mathrm{H}_{1.1}$ & Hay vínculo entre sentirse cartagenero y el sentido de identidad. \\
\hline $\mathrm{H}_{1.2}$ & $\begin{array}{l}\text { Hay vínculo entre sentirse cartagenero y ser sujetos de derechos como } \\
\text { negritudes. }\end{array}$ \\
\hline $\mathrm{H}_{1.3}$ & Hay vínculo entre sentirse cartagenero y la participación comunitaria. \\
\hline $\mathrm{H}_{1.4}$ & $\begin{array}{l}\text { Hay vínculo entre sentirse cartagenero y la gestión sobre el territorio por } \\
\text { parte de los líderes comunitarios. }\end{array}$ \\
\hline $\mathrm{H}_{1.5}$ & Hay vínculo entre sentirse cartagenero y la valoración de la cultura afro. \\
\hline $\mathrm{H}_{1.6}$ & Hay vínculo entre sentirse cartagenero y la participación democrática. \\
\hline
\end{tabular}

Tabla 4: Resumen de procesamiento de casos del modelo

\begin{tabular}{|l|l|c|c|}
\hline \multicolumn{2}{|c|}{ Casos sin ponderar ${ }^{a}$} & $N$ & Porcentaje \\
\hline \multirow{4}{*}{ Casos seleccionados } & Incluido en el análisis & 96 & 99 \\
\cline { 2 - 4 } & Casos perdidos & 1 & 1 \\
\cline { 2 - 4 } & Total & 97 & 100 \\
\hline Casos no seleccionados & 0 & 0 \\
\hline Total & 97 & 100 \\
\hline \multicolumn{3}{|c|}{ Codificación de variable dependiente } \\
\hline Valor original & 0 \\
\hline Sí & 1 \\
\hline No & \multicolumn{3}{|c|}{0} \\
\hline
\end{tabular}

Como resultado se obtiene que los casos incluidos en el análisis responden al 99\% de los datos, con la siguiente codificación, variable en valor de 0 para representar que sí se siente cartagenero y en 1 no se siente ciudadano. Así mismo, el 86,5\% indica el número de casos que el modelo es capaz de predecir correctamente en la ecuación de regresión logística y los datos observados en las variables independientes, Por lo tanto, el modelo es explicativo porque alcanza el $86,5 \%>50 \%$ de los casos de estudio; ver tabla 5 .

Tabla 5: Tabla de clasificación de observaciones del modelo. a. El valor de corte es ,500

\begin{tabular}{|c|c|c|c|c|}
\hline \multirow{2}{*}{ Observado } & \multicolumn{3}{|c|}{ Pronosticado } \\
\cline { 3 - 5 } \multicolumn{2}{|c|}{} & Ciudadano de Cartagena de Indias & \multirow{2}{*}{ Porcentaje correcto } \\
\cline { 2 - 5 } & Sí & No & \\
\hline \multirow{2}{*}{ Ciudadanía } & Sí & 76 & 3 & 96,2 \\
\cline { 2 - 5 } & No & 10 & 7 & 41,2 \\
\hline \multicolumn{2}{|l|}{ Porcentaje global } & & & 86,5 \\
\hline
\end{tabular}

En la Tabla 6 se aprecia la Puntuación de Eficiencia Estadística de ROA (Chi-cuadrado: 24,575; gl: 6; Sig < 0,05 ) es de 0,00 , por lo cual el modelo ayuda a explicar el evento, es decir las variables independientes explican la ocurrencia de la variable dependiente. Por lo cual se rechaza la hipótesis nula. Considerando el valor de la R-cuadrado de Nagelkerke se concluye que el conjunto de variables independientes explica un $37,2 \%$ de la varianza de la variable dependiente.

Tabla 6: Pruebas ómnibus de coeficientes de modelo (método entrar). La estimación ha terminado en el número de iteración 6 porque las estimaciones de parámetro han cambiado en menos de ,001.

\begin{tabular}{|l|c|c|c|}
\cline { 2 - 4 } \multicolumn{1}{c|}{} & Chi-cuadrado & Gl & Sig. \\
\hline Paso & 24,575 & 6 & 0 \\
\hline Bloque & 24,575 & 6 & 0 \\
\hline Modelo & 24,575 & 6 & 0 \\
\hline Paso & Log de la verosimilitud -2 & R cuadrado de Cox y Snell & R cuadrado de Nagelkerke \\
\hline 1 & $65,077 a$ & 0,226 & 0,372 \\
\hline
\end{tabular}


La fuerza de las relaciones entre variables viene expresada en los exponentes de los coeficientes de la regresión $\operatorname{Exp}(B)$. En la tabla 7 se recoge la información del modelo. Los resultados finales permiten concluir que las variables independientes que explican el modelo son: la participación a través de ser miembro de Consejo Comunitario, la gestión de los líderes comunitarios y la valoración cultural, cuya significatividad muestra un valor menor de 0,05 , por lo tanto, tienen alta probabilidad de acierto en la predicción del sentimiento de ciudadanía; por lo cual se validan las siguientes subhipotesis: i) $\mathrm{H}_{1.3}$ Hay vínculo entre sentirse cartagenero y la participación en el Consejo comunitario; ii) $\mathrm{H}_{1.4}$ Hay vínculo entre sentirse cartagenero y la gestión sobre el territorio por parte de los líderes comunitarios; y iii) $\mathrm{H}_{1.5}$ Hay vínculo entre sentirse cartagenero y la valoración de la cultura afro.

Tabla 7: Variables en la ecuación de regresión logística.

\begin{tabular}{|c|c|c|c|c|c|c|}
\hline & $B$ & Error estándar & Wald & Gl & Sig. & $\operatorname{Exp}(B)$ \\
\hline Identidad afrodescendiente & 0,136 & 0,103 & 1,747 & 1 & 0,186 & 1,146 \\
\hline Protección a los derechos de la comunidad afrodescendiente & $-0,019$ & 0,235 & 0,006 & 1 & 0,937 & 0,982 \\
\hline Participación comunitaria & 0,345 & 0,125 & 7,605 & 1 & 0,006 & 1,412 \\
\hline Gestión del liderazgo Comunitario & $-0,67$ & 0,201 & 11,078 & 1 & 0,001 & 0,511 \\
\hline Valoración y respeto a la cultura afro descendiente & $-0,479$ & 0,236 & 4,112 & 1 & 0,043 & 0,619 \\
\hline Participación democrática & 0,175 & 0,189 & 0,86 & 1 & 0,354 & 1,192 \\
\hline Constante & 0,81 & 1,176 & 0,474 & 1 & 0,491 & 2,249 \\
\hline
\end{tabular}

Las demás variables dependientes incluida en el modelo no son significativas, contemplando las subhipotesis nulas que indican que no existen dependencia entre: sentirse cartagenero y la participación democrática $\mathrm{H}_{1.6}$, sentirse cartagenero y el sentido de identidad $\mathrm{H}_{1.1}$ y sentirse cartagenero y ser sujetos de derechos como negritudes $\mathrm{H}_{1.2}$. Al interpretar los resultados de $\operatorname{Exp} \beta$ se establece una relación de fortaleza entre "formar parte de Consejo Comunitario" y ser "ciudadano de Cartagena de Indias" la probabilidad de que se presente sentirse ciudadano es 1,412 veces más para la persona que forma parte de esta colectividad. Bustillo (2020)

\section{CONCLUSIONES}

De acuerdo al estudio, discusión y análisis de esta investigación se pueden extraer las siguientes conclusiones principales:

1) Aun existiendo un abandono evidente por parte de los entes gubernamentales y las inequidades latentes, las comunidades gestionan su propio desarrollo desde la participación en los consejos comunitarios de sus territorios, creando una modalidad de agenda comunitaria para su desarrollo, De otra parte se pudo comprobar que para la poblaciones de minorías étnicas afro descendientes ser miembro del Consejo Comunitario da una mayor probabilidad de sentirse incluido en la dinámica territorial, de sentirse ciudadano.

2) Cuando se siente la buena gestión de los líderes en la defensa de los derechos de la comunidad afrodescendiente y del territorio ancestral con todos sus recursos naturales y culturales, nace el empoderamiento de toda la comunidad utilizando los mecanismos para la exigencia de derechos como ciudadanos, desde las dinámicas grupales de capacitación, reivindicación, manifestaciones colectivas y exigencias legales.

3) Las personas que valoran su cultura ancestral, respetan su manifestaciones y formas de vida afro, sus procesos de transformación histórica, sus luchas frente a la equidad, sienten ser ciudadanos de Cartagena de Indias, se visibilizan al incluirse en el mapa poblacional de la ciudad, se identifican con el cartagenero común y conforman el sentido colectivo de ciudadanía.

\section{AGRADECIMIENTOS}

Los autores expresan su agradecimiento a la Universidad de Castilla - La Mancha de España, por todo su apoyo a la tesis doctoral titulada, la Responsabilidad Social Empresarial a través de la legitimidad social. Valoración de la aplicación de la Ley 21 de 1991 de consultas previas en el Caribe Colombiano, de la cual deriva el presente artículo científico.

\section{REFERENCIAS}

Ayala-García, J., y Meisel-Roca, A. Cartagena Libre de Pobreza Extrema en el 2033, Documentos de Trabajo sobre Economía Regional y Urbana; no. 257 (2017)

Barnham, C., Quantitative and Qualitative Research: Perceptual Foundations, https://doi.org/10.2501//JMR-2015-070, International Journal of Market Research, 57(6), 837-854 (2015)

Barreira, C., Tavares, J., y otros tres autores. Conflictos Sociales, Luchas Sociales y Políticas de Seguridad Ciudadana, 1a ed., Ciudad de México D.F, México (2013) 
Bherer, L., Dufour, P., y Montambeault, F., The Participatory Democracy Turn: An Introduction, https://doi.org/10.1080/17448689.2016.1216383, Journal of Civil Society, 12 (3) 225-230 (2016)

Bolondi, G., Branchetti, L., y Giberti, C. A., Quantitative Methodology for Analyzing the Impact of the Formulation of a Mathematical Item on Students Learning Assessment, https://doi.org/10.1080/01900692.2016.1263659, Studies in Educational Evaluation, 58, 37-50 (2018)

Bustillo, C. M. La Responsabilidad Social Empresarial a Través de la Legitimidad Social. Valoración de la Aplicación de la Ley 21 de 1991 de Consultas Previas en el Caribe Colombiano. Universidad de Castilla - La Mancha, España (2020)

Campos-Carreño, M. L., Velasco, C. B., y Araya, J. P., Adaptación y Validación de Escalas de Medición en el Trabajo. Parte 1: Bienestar Social, Información Tecnológica, 31(5), 195-204 (2020)

Chetty, S., The Case Study Method for Research in Small-and Medium-sized Firms,

https://doi.org/10.1177/0266242696151005, International Small Business Journal, 15(1), $73-85$ (1996)

Eisenhardt, K. M., Building Theories from Case Study Research, https://doi.org/10.2307/258557, Academy of Management Review, 14(4), 532-550 (1989)

Espinosa-Espinosa, A., Madero-Jirado, M., y otros dos autores. Etnicidad, Espacio y Desarrollo Humano en Comunidades Pobres Urbanas: la Comuna 6 en Cartagena de Indias, Colombia, https://doi.org/10.15446/cuad.econ.v39n81.77333, Cuadernos de Economía, 39(81), 635-665 (2020)

Fonseca, X., Lukosch, S., y Brazier. F. Social Cohesion Revisited: a New Definition and How to Characterize it, Innovation: The European Journal of Social Science Research, 32(2), 231-253 (2018)

Graizbord, B., Planeación Urbana, Participación Ciudadana y Cambio Social, https://doi.org/10.22136/est001999461, Economía, Sociedad y Territorio, 2(5), 149-161 (1999)

Harrison, H., Birks, M., y otros dos autores. Case Study Research: Foundations and Methodological Orientations, https://doi.org/10.17169/fqs-18.1.2655, Forum Qualitative Sozialforschung, 18(1) 17 (2017)

Hooker, J., Afro-descendant Struggles for Collective Rights in Latin America: Between Race and Culture, https://doi.org/10.1080/10999940802347764, Souls, 10(3), 279-291 (2008)

Hyland, M., y Bertsch, V., The Role of Community Involvement Mechanisms in Reducing Resistance to Energy Infrastructure Development, https://doi.org/10.1016/j.ecolecon.2017.11.016, Ecological Economics, 146, 447-474 (2018)

Lennox, C., y Minott, C., Inclusion of Afro-Descendents in Ethnic Data Collection: Towards Visibility, International Journal on Minority and Group Rights, 18(2), 257-275 (2011)

Lever, J., Krzywinski, M., y Altman N., Logistic regression, https://doi.org/10.1038/nmeth.3904, Nature Methods, 13, 541-542 (2016)

Mansournia, M. A., Geroldinger, A., y otros dos autores. Separation in Logistic Regression: Causes, Consequences, and Control, https://doi.org/10.1093/aje/kwx299, American Journal of Epidemiology, 187(4), 864-870 (2018)

Martiskainen, M., The Role of Community Leadership in the Development of Grassroots Innovations, https://doi.org/10.1016/j.eist.2016.05.002, Environmental Innovation and Societal Transitions, 22, 78-89 (2017)

McNeish, J. A., A Vote to Derail Extraction: Popular Consultation and Resource Sovereignty in Tolima, Colombia, https://doi.org/10.1080/01436597.2017.1283980, Third World Quarterly, 38(5), 1128-1145 (2017)

Meredith, J. R., Raturi, A., K., y otros dos autores. Alternative Research Paradigms in Operations, https://doi.org/10.1016/0272-6963(89)90033-8, Journal of Operations Management, 8(4), 297-326 (1989)

Michels, A., y De Graaf, L., Examining Citizen Participation: Local Participatory Policymaking and Democracy Revisited, https://doi.org/10.1080/03003930.2017.1365712, Local Government Studies, 43(6), 875-881 (2017)

Moses, J., Social citizenship and Social Rights in an Age of Extremes: TH Marshall's Social Philosophy in the Longue Durée, https://doi.org/10.1017/S1479244317000178, Modern Intellectual History, 16(1), 155-184 (2019)

Olivo, M. G. Educación Para la Ciudadanía en Chile, Información Tecnológica, 28 (5),151-164 (2017)

Ortiz, L., La Instancia Pública de la Gestión. Algunas Consideraciones Sobre la Participación Ciudadana en el Estado, Rev. del CLAD Reforma y Democracia, (54), 179-194 (2012)

Schick-Makaroff, K., MacDonald, M., y otros tres autores. What Synthesis Methodology Should I use? A Review and Analysis of Approaches to Research Synthesis, https://doi.org/10.3934/publichealth.2016.1.172, AIMS Public Health, 3(1), 172-215 (2016)

Wirtz, B. W., Weyerer, J. C., y Rösch, M., Citizen and Open Government: An Empirical Analysis of Antecedents of Open Government Data, https://doi.org/10.1080/01900692.2016.1263659, International Journal of Public Administration, 41(4), 308-320 (2018)

Xu, K., Zhang, J., y Tian, F. Community Leadership in Rural Tourism Development: A Tale of Two Ancient Chinese Villages, Sustainability, 9(12), 2344 (2017)

Yin, R. K., Validity and Generalization in Future Case Study Evaluations, https://doi.org/10.1177/1356389013497081, Evaluation, 19(3), 321-332 (2013) 